\title{
PMWS: experimental model and co-infections
}

\author{
G.M. Allan ${ }^{\text {a,* }}$, F. McNeilly ${ }^{\text {a }}$, J. Ellis ${ }^{\text {b }}$, S. Krakowka ${ }^{\text {c }}$, A. Botner ${ }^{\text {d }}$, \\ K. McCullough ${ }^{\mathrm{e}}$, H. Nauwynck ${ }^{\mathrm{f}}$, S. Kennedy ${ }^{\mathrm{a}}$, \\ B. Meehan ${ }^{\mathrm{a}}$, C. Charreyre ${ }^{\mathrm{g}}$ \\ a Virology Department, Veterinary Research Laboratory, Veterinary Sciences Division, Stoney Road Stormont, \\ DARDNI, Stormont, BT4 3SD Belfast, Northern Ireland, UK \\ ${ }^{\mathrm{b}}$ Department of Veterinary Microbiology, University of Saskatchewan, Sask., Canada \\ c Ohio State University, OH, USA \\ d Danish Veterinary Institute for Virus Research, Lindholm, Denmark \\ ${ }^{\mathrm{e}}$ Institute for Virology and Immunoprophylaxix, Mittelhausern, Switzerland \\ ${ }^{\mathrm{f}}$ Virology Laboratory, University of Gent, Gent, Belgium \\ ${ }^{g}$ Merial SAS, Lyon, France
}

\begin{abstract}
Porcine circovirus type 2 (PCV2) is now recognised as the causal agent of porcine multisystemic wasting syndrome (PMWS), an economically important wasting disease of young pigs [J. Vet. Diagn. Invest. 12 (2000) 3]. Gross lesions of PMWS include generalised lymphadenopathy, hepatitis, nephritis and pneumonia and typical histological lesions include lymphocytic depletion and multinucleated giant cell formation in lymph nodes, degeneration and necrosis of hepatocytes, and multifocal lymphohistocytic interstitial pneumonia.

This communication will review the results of experimental infections of gnotobiotic (GN), colostrum-deprived (CD) and colostrum-fed (CF) pigs within our group, and elsewhere, with PCV2 and the conclusions that can be drawn from this work. (C) 2003 Elsevier B.V. All rights reserved.
\end{abstract}

Keywords: Porcine; Circovirus; Experimental infection; Wasting

\section{Experimental infections of GN piglets}

The us of GN piglets in studying the pathogenesis of "new" or "emerging" viral infections offers a model to researchers that cannot be influenced by other infectious agents and external co-factors. This "pure" model has been used within our group

\footnotetext{
* Corresponding author. Tel.: +44-1232-525679; fax: +44-1232-525750.

E-mail address: gordon.allan@dardni.gov.uk (G.M. Allan).
}

to study the pathogenesis of PCV2 infections (Ellis et al., 1999; Krakowka et al., 2000, 2001). The first demonstration of experimental reproduction of lesions consistent with PMWS following inoculation with PCV2 was carried out in 1997 in GN piglets (Ellis et al., 1999). Unfortunately (or fortunately) some of the PCV2 inoculum used in this study was contaminated with porcine parvovirus (PPV), leading to speculation that PCV2 alone was not capable of producing lesions consistent with field disease. 
In a second study (Krakowka et al., 2000), highly purified, cell culture grown isolates of PCV1, PCV2 and PPV were used individually, or in combination, to infect GN piglets. Piglets infected with PCV2 alone did not develop clinical disease but had mild lymphoplasmacytic hepatitis and cholangiohepatitis. However piglets co-infected with PCV2 and PPV developed severe clinical disease and gross lesions of PMWS. An abundance of PCV2 antigen was associated with lesions. The primary lesion in these animals was widespread disseminated angiocentric granulomatous inflammation. Piglets infected with PPV or PCV1 alone or co-infected with PCV1 and PPV did not develop clinical disease and did not have significant gross or histological lesions.

The authors concluded from these results that PCV2 was essential to cause PMWS in GN pigs and the mechanisms that accounts for the synergistic relationship between PCV2 and PPV demonstrated in their GN model remained elusive.

A third set of experiments (Krakowka et al., 2001) have gone some way towards elucidating this mechanism of enhanced PCV2 replication, leading to disease. In this study, GN pigs were infected with PCV2 alone and essentially divided into two groups (and controls). One group was systemically immunostimulated by injection of keyhole limpet haemocyanin in incomplete Freund's adjuvant (KLH/ICFA) in four sites: axillas and hips. The second group was not immunostimulated. All pigs given PCV2 alone and immunostimulated (7 of 7) developed clinical disease and had gross and histological lesions consistent with PMWS. None of the pigs given PCV2 alone without immunostimulation developed clinical disease and histological lesions consistent with PMWS in these piglets were mild. The authors concluded that in their GN model activation of the immune system and infection with PCV2 are the pivotal events in the development of clinical PMWS. It is interesting to note that, to date, this GN immunostimulation model is the only mechanism to produce $100 \%$ hit rate for experimentally reproduced PMWS using a single infectious agent.

A recent experiment in GN pigs has demonstrated that PCV2 infection of pigs that were immunosupressed using cyclosporine and/or the steroid triamcinolone acetonide failed to consistently produce PMWS. In this experiment only 1 of 8 immunosu- pressed piglets developed disease compared with 4 of 4 immunostimulated controls.

Clearly, these experimental infections of GN pigs have been of major importance in understanding the pathogenic process of PCV2 infections and PMWS.

\section{Experimental infection of CD pigs}

The first report of experimental reproduction of PMWS in this model (Allan et al., 1999) confirmed the results of Ellis et al. (1999) in GN pigs, in that co-infection of CD pigs with PCV2 and PPV resulted in clinical disease and gross and histological lesions in all inoculates. Again, histological lesions were associated with an abundance of PCV2 antigen and minimal amounts of PPV antigen. However, in contrast to the results in GN pigs, in the CD model, 1 of 4 piglets inoculated with PCV2 alone developed PMWS and severe histological lesions. The remaining three inoculates were clinically normal with mild to moderate histological lesions. Other workers have reported that experimental infection of $\mathrm{CD} / \mathrm{CD}$ pigs with PCV2 alone produced severe clinical disease in 6 of 23 inoculates with histological lesions consistent with PMWS (Bolin et al., 2001), confirming the findings of Allan et al. (1999).

In a second experiment in CD pigs, Allan et al. (2000) demonstrated that co-infection with PCV2 and PRRSV potentiated PCV2 replication, leading to increased viral load in tissues and more severe histological lesions. Histological lesions were again associated with PCV2 with little or no PRRV antigen demonstrated. Clinical disease was not reproduced in these inoculates although the production of severe clinical disease following experimental co-infection of CD/CD pigs with PCV2 and PRRSV has been reported (Harms et al., 2001). In this experiment a PCV2 and a PRRSV isolate from cases of sow abortion and mortality syndrome (SAMS) were used to infect 3 -week-old $\mathrm{CD} / \mathrm{CD}$ piglets. The piglets inoculated with PRRSV alone had a transient, moderate respiratory disease, with no deaths while 5 of 13 piglets inoculated with PCV2 alone died within 10 days PI. In the same experiment 10 of 11 piglets inoculated with PCV2 and PRRSV died within this time scale. These results indicate that the PCV2 isolated from SAMS may have been the predominant pathogen in 
this disease and emphasises the importance of PCV2 as a pathogen of swine.

\section{Experimental infection of CF pigs}

A number of studies on experimental infection of CF pigs with PCV2 have been reported. Magar et al. (2000) reported no major clinical signs and limited gross lesions in 3-4-week-old seronegative pigs inoculated with PCV2. However, mild to moderate histological lesions consistent with PMWS were demonstrated in inoculates. Balasch et al. (1999) also failed to produce clinical disease in 8-week-old CF inoculates using a tissue homogenate from a PCV2 infected pig, but again, did produce histological lesions consistent with PMWS. In contrast, Reynaud et al. (2000) has demonstrated clinical signs of disease in $69 \%$ of 7 -week-old CF pigs inoculated with PCV2 and clinical wasting and death in 2/16 (12.5\%) inoculates.

Recently, inoculation of seronegative SPF piglets with PCV2 has produced clinical disease in 4 of 10 inoculates (Ladekjaer-Mikkelsen et al., 2002). In this experiment 3-week-old PCV2 seronegative pigs were inoculated with PCV2, with and without immunostimulation with KLH/ICF. Three of 5 inoculates in the non-stimulated group developed PMWS but only 1 of 5 in the stimulated group. These results would appear to contradict the findings of Krakowka et al. (2001). However, it is important to note that these animals were not held in biologically secure units and could have been exposed to other immunostimulatory events during the experiment. Further studies in CF pigs are required.

Four-week-old SPF pigs have also been used in experimental studies with a PCV2 inoculum derived from a molecular clone constructed by ligating the complete PCV2 genome in tandem into a pSK vector (Halbur, 2001). Forty pigs were randomly assigned into four groups of 10 each. Following experimental infection, gross and microscopic lesions consistent with PMWS were seen in lymphoid tissues and lungs of pigs in groups inoculated with the homogeneous PCV2 live stock derived from the molecular clone and propagated in cell culture. This experiment has provided conclusive prove that infection of pigs with PCV2 alone can result in lesions consistent with PMWS.

\section{Conclusions}

Experimental inoculation of GN, CD and CF piglets with PCV2 alone has produced clinical PMWS and it is now accepted that PCV2 is the causal agent of this disease. However, it appears that the severity of disease the can be increased if GN and/or CD pigs are co-infected with other agents or immunostimulated. The mechanism by which this increased severity of disease is potentiated has yet to be elucidated, but it is likely that the up regulation of some component of the porcine immune system provides "actively dividing cells" for increased replication of PCV2 virus. Limited studies on PCV2 infection of seronegative, CF pigs indicates that immunostimulation may not be necessary in this model for the development of PMWS, following PCV2 infection, however, further studies are required in this area.

One question still remains to be answered. PCV2 is not a "new" virus and PMWS is not a "new" disease as retrospective studies have identified PCV2-associated cases of PMWS from 1986. Indeed, a recently completed study within our group has shown that a PCV2 isolate from non-diseased pig from a non-PMWS pig herd that was isolated in 1993 can cause severe PMWS when inoculated into CD pigs. Why then has a PMWS epidemic exploded global in the last 3-4 years? Is it possible that changes in the global pig industry have resulted in up-regulating a common sub-clinical PCV2 infection to PMWS?

\section{Acknowledgements}

Some areas of this work were funded by Merial SAS and by the European Union (project No. GLK2-CT-1999-00445).

\section{References}

Allan, G.M., Kennedy, S., McNeilly, F., Foster, C., Ellis, J.A., Krakowka, S.J., Meehan, B.M., Adair, B.M., 1999. Experimental reproduction of wasting disease and death by co-infection of pigs with PCV2 and PPV. J. Comp. Pathol. $121,1-11$.

Allan, G.M., McNeilly, F., Ellis, J., Krakowka, S., Meehan, B., McNair, I., Walker, I., Kennedy, S., 2000. Experimental infection of CD piglets with PCV2 and PRRSV potentiates PCV2 replication. Arch. Virol. 145, 2421-2429. 
Balasch, M., Segalis, J., Rosell, C., Domingo, M., Mankertz, A., Urniza, A., Plana-Duran, J., 1999. Experimental inoculation of conventional pigs with tissue homogenates from pigs with PMWS. J. Comp. Pathol. 121, 139-148.

Bolin, S.R., Stoffregen, W.C., Nayar, G.P.S., Hamel, A.L., 2001. Postweaning multisystemic wasting syndrome induced after experimental inoculation of $\mathrm{CD} / \mathrm{CD}$ piglets with type 2 porcine circovirus. J. Vet. Diagn. Invest. 13, 185-194.

Ellis, J., Krakowka, S., Larimore, M., Hains, D., Bratanich, A., Clark, E., Allan, G.M., Konoby, C., Hassard, L., Meehan, B., Martin, K., Harding, J., Kennedy, S., McNeilly, F., 1999. Reproduction of lesions of postweaning multisystemic wasting syndrome in gnotobiotic pigs. J. Vet. Diagn. Invest. 11, 3-14.

Halbur, P., 2001. PMWS: Improved models and recent findings of clinical significance. In: Proceedings of the Ninth Swine Disease Conference for Swine Practitioners, November 2-9. Iowa State University, Iowa, pp. 162-166.

Harms, P.A., Sorden, S.D., Halbur, P.G., Bolin, S.R., Lager, K.M., Morozov, I., Paul, P.S., 2001. Experimental reproduction of severe disease in $\mathrm{CD} / \mathrm{CD}$ pigs concurrently infected with type 2 porcine circovirus and porcine reproductive and respiratory syndrome virus. Vet. Pathol. 38, 528-539.
Krakowka, S., Ellis, J.A., Meehan, B., Kennedy, S., McNeilly, F., Allan, G.M., 2000. Viral wasting syndrome of swine: experimental reproduction of PMWS in gnotobiotic swine by co-infection with PCV2 and PPV. Vet. Pathol. 37, 254-263.

Krakowka, S., Ellis, J.A., McNeilly, F., Ringler, S., Rings, D.M., Allan, G., 2001. Activation of the immune system is the pivotal event in the production of wasting disease in pigs infected with PCV2. Vet. Pathol. 38, 31-42.

Ladekjaer-Mikkelsen, A.S., Nielsen, J., Stadejek, T., Storgaard, T., Krakowka, S., Ellis, J., McNeilly, F., Allan, G., Botner, A., 2002. Reproduction of postweaning multisystemic wasting syndrome (PMWS) in immunostimulated and non-immunostimulated 3-week-old piglets experimentally infected with porcine circovirus type 2 (PCV2). Vet. Microbiol. 89 (23), 97-114.

Magar, R., Larochelle, R., Thibault, S., Lamontagne, L., 2000. Experimental transmission of PCV2 in weaned pigs: a sequential study. J. Comp. Pathol. 123, 258-269.

Reynaud, G., Boeuf, L., Brunet, S., Charreyre, C., 2000. Experimental reproduction of PMWS by PCV2 challenge in piglets aged 7 weeks. In: Proceedings of the 16th International Pig Veterinary Society Congress, Melbourne, Australia, p. 578. 